\title{
ACIDENTES DE TRABALHO: ANÁLISES ESTATÍSTICAS NA AGRICULTURA, INDÚSTRIA E CONSTRUÇÃO CIVIL ${ }^{1}$
}

\author{
Ketelin Rúbia Almeida Silva² \\ Júlia Silva de Jesus \\ André Henrique Garcia Silva \\ Talita Emidio Andrade Soares \\ Denilson Junio Marques Soares
}

\begin{abstract}
RESUMO
Quando um acidente ocorre no local e no tempo de trabalho ele é chamado de acidente de trabalho, que pode ser ocasionado por diversos fatores como fadiga, estresse, uso de materiais perigosos ou mesmo por imprudências do empregador ou do empregado. Estudos indicam a existência de atividades mais propensas a este tipo de acidente e que a prevenção, a conscientização e o treinamento dos trabalhadores são fatores essenciais para a sua redução. $\mathrm{O}$ objetivo deste trabalho foi verificar se existem diferenças estatisticamente significativas entre as médias de acidentes de trabalho de três expressivos grupamentos de atividades: agricultura, indústria e construção civil. Trata-se de um estudo analítico-descritivo, produzido a partir de dados quantitativos obtidos em bases de dados nacionais, oriundas do Sistema Federal de Inspeção do Trabalho e referentes aos anos compreendidos entre 2010 e 2016. Observou-se que a construção civil apresentou uma média de acidentes superior às demais e que as médias obtidas pela agricultura e indústria não apresentaram diferenças à 5\% de nível de significância. Espera-se que este trabalho sirva como um instrumento de difusão e alerta aos perigos que a falta de investimento em segurança do trabalho pode ocasionar, especialmente no campo da construção civil.
\end{abstract}

Palavras-chave: Acidentes de trabalho. Segurança do trabalho. Testes de hipóteses. Construção civil.

\section{ACCIDENTS AT WORK: STATISTICAL ANALYSIS ON AGRICULTURE, INDUSTRY AND CONSTRUCTION}

\begin{abstract}
When an accident occurs in the workplace and during work hours it is called an accident at work, which can be occasioned by several factors such as fatigue, stress, use of hazardous materials or even by imprudences of the employer or the employee. Studies indicate that there are activities that are more prone to accidents and that prevention, awareness and training of

${ }^{1} 1$ Como citar este artigo:

SILVA, K. R. A. et al. Acidentes de trabalho: análises estatísticas na agricultura, indústria e construção civil. ForScience, Formiga, v. 8, n. 2, e00729, jul./dez. 2020. DOI: 10.29069/forscience.2020v8n2.e729.
\end{abstract}

${ }^{2}$ Autor para correspondência: Ketelin Rúbia Almeida Silva, e-mail: ketelin.rubia.almeida@gmail.com. 
workers are essential factors in reducing these eventualities. The objective of this study was to verify if there are significant differences between the averages of accidents at work in three expressive groups of activities: agriculture, industry and construction through an analyticaldescriptive study, produced from quantitative data obtained in national databases, from the Federal System of Labor Inspection and referring to the years 2010 to 2016. It was observed that the construction presented na average of accidents higher than the others and that the averages obtained by agriculture and industry did not show differences at $5 \%$ of significance. It is expected that this work can be used as an instrument of diffusion and alert to the dangers the lack of investment in work safety may cause, especially in the field of construction.

Keywords: Accidents at work. Workplace safety. Statistical hypothesis testing. Construction.

\section{INTRODUÇÃO}

De acordo com o Centro de Estudos da Saúde do Trabalhador e Ecologia Humana, o Brasil é o quarto país com maiores registros de mortes por atividades laborais atrás, apenas, dos Estados Unidos, da Tailândia e da China. Quando se trata de acidentes de trabalho somos o quinto, atrás da Colômbia, França, Alemanha e dos Estados Unidos (CESTEH, 2019).

No ano de 2017, foram contabilizados mais de 574 mil acidentes e quase 2 mil mortes (CESTEH, 2019). Estes índices são, em muitos casos, consequência das péssimas condições de trabalho oferecidas, da falta de equipamentos de segurança e de longas jornadas de trabalho. Alguns empregadores veem a adoção de medidas preventivas como gastos desnecessários, assumindo riscos que podem ceifar a vida de seus funcionários.

O Art. 19 da Lei $\mathrm{n}^{\circ}$ 8.213, de 24 de julho de 1991 que dispõe sobre os Planos de Benefícios da Previdência Social e dá outras providências, define acidente de trabalho como

[...] o que ocorre pelo exercício do trabalho a serviço da empresa ou pelo exercício do trabalho dos segurados [...], provocando lesão corporal ou perturbação funcional que cause a morte ou a perda ou redução, permanente ou temporária, da capacidade para o trabalho (BRASIL, 1991).

Uma cultura de segurança no trabalho estabelecida pode contribuir significativamente para evitar esses acidentes e, consequentemente, para um aumento na produção e economia das empresas, pois além de reduzir (ou eliminar) os riscos de acidentes de trabalho, tem impactos na produtividade, garantindo qualidade de vida dentro do ambiente de trabalho.

Chiavenato (2002) pressupõe que:

[...] segurança no trabalho é o conjunto de medidas técnicas, educacionais, médicas e psicológicas empregadas para prevenir acidentes, quer eliminando as condições inseguras do ambiente quer instruindo ou convencendo as pessoas da implantação de práticas preventivas (CHIAVENATO, 2002, p.438). 
Embora seja um tema largamente abordado e disseminado em todo o mundo, é necessário que a segurança no trabalho esteja frequentemente em pauta de discussões e na rotina de qualquer empresa que visa a responsabilidade social e o bem-estar dos seus funcionários e familiares, considerando que, segundo Oliveira (1998), não se pode obter uma qualidade de vida sem se obter a qualidade no trabalho.

Entre os setores com as maiores taxas de acidentes de trabalho destacam-se a indústria, a agricultura e a construção civil. Em termos gerais, a indústria é o setor responsável por transformar matéria-prima em produtos comercializáveis. Para isto, é frequente o uso da força humana, de maquinários e da energia, responsáveis pela maioria dos acidentes de trabalho no setor.

O setor agrícola é uma das bases de sustentação da economia brasileira desde os primórdios da colonização. O avanço tecnológico deste e, consequentemente, a expansão da fronteira agrícola elevaram os índices de acidente de trabalho, fazendo com que o Ministério do Trabalho e Emprego reconhecesse, especialmente a agropecuária, como de "alto risco para a segurança e saúde dos trabalhadores" (SOUSA; SANTANA, 2015, p. 2).

O setor da construção civil também apresenta elevados índices de irregularidades, considerando a segurança de seus trabalhadores,

[...] no ambiente da Construção Civil, existem inúmeras situações de risco capazes de provocar acidentes de trabalho. Assim, a análise de fatores de risco em todas as atividades e operações é fundamental para a prevenção de ocorrências. Entre os fatores de risco que podem provocar acidentes de trabalho, destacam-se: máquinas, equipamentos e ferramentas; eletricidade; incêndio; armazenamento e transporte de materiais; manuseio de produtos perigosos; queda em altura, entre outros (COELHO; GHISI 2016, p. 27).

Dessa forma, este artigo tem o objetivo de propor um debate sobre os alarmantes índices de acidentes de trabalho nestes três expressivos setores da economia e verificar se existem diferenças estatisticamente significativas entre as médias desses acidentes nestes setores.

\section{REFERENCIAL TEÓRICO}

Um problema comum em experimentos estatísticos é comparar tratamentos na busca por algum que possua média diferente dos demais (superior ou inferior). Esta tarefa pode ser de difícil execução quando os valores encontrados forem próximos, não sendo possível decidir, subjetivamente, se as diferenças existentes são reais ou consequência de alguma fonte de variação, como erros causados pela aleatoriedade. 
O método estatístico comumente utilizado nestes tipos de experimentos é a Análise de Variância (ANOVA). Trata-se de uma técnica estatística capaz de verificar se existe diferença significativa entre as médias dos tratamentos e se os fatores exercem influência em alguma variável dependente.

Dessa forma, a hipótese de nulidade $\left(H_{0}\right)$ testada na ANOVA é a de que as médias populacionais são iguais. Como hipótese alternativa $\left(H_{1}\right)$, temos que pelo menos uma das médias em análise é diferente das demais. A ANOVA retorna uma estatística $F$ para avaliar o tamanho desta diferença. Para que a hipótese de nulidade não seja rejeitada, espera-se que o valor desta estatística esteja próximo de 1.

A Tabela 1 indica como a ANOVA é organizada e como a estatística $F$ é determinada.

Tabela 1 - Análise de variância

\begin{tabular}{ccccc}
\hline $\begin{array}{c}\text { Fonte de } \\
\text { Variação }\end{array}$ & $\begin{array}{c}\text { Graus de } \\
\text { Liberdade }\end{array}$ & $\begin{array}{c}\text { Soma de } \\
\text { Quadrados }\end{array}$ & $\begin{array}{c}\text { Quadrado } \\
\text { Médio }\end{array}$ & F \\
\hline $\begin{array}{c}\text { Entre } \\
\text { os grupos }\end{array}$ & $k-1$ & $S Q_{E}=\sum_{i=1}^{k} n_{i}\left(\bar{y}_{i}-\bar{y}\right)^{2}$ & $Q M_{E}=\frac{S Q_{E}}{k-1}$ & $\frac{Q M_{E}}{Q M_{D}}$ \\
$\begin{array}{c}\text { Dentro } \\
\text { dos grupos }\end{array}$ & $n-k$ & $S Q_{D}=\sum_{i=1}^{k}\left(n_{i}-1\right) s_{i}{ }^{2}$ & $Q M_{D}=\frac{S Q_{D}}{n-k}$ & \\
Total & $n-1$ & & & \\
\hline
\end{tabular}

Fonte: Bussab e Morettin (2013).

Para executar a ANOVA, alguns pressupostos básicos precisam ser atendidos: as amostras devem ser aleatórias e independentes; as populações devem ter distribuição normal e as variâncias populacionais são homogêneas (BUSSAB; MORETTIN, 2013).

O pressuposto da independência pode ser avaliado por meio da análise dos resíduos do modelo ANOVA e quando não há um padrão no comportamento gráfico dos resíduos, considera-se, subjetivamente, que o pressuposto foi atendido (CECON et al., 2012).

Para verificar se as populações têm distribuição normal pode-se aplicar o teste de Kolmogorov-Smirnov, cuja hipótese de nulidade é a de que os dados seguem uma distribuição normal. Em suma, este teste observa o valor máximo obtido pelo módulo da diferença entre a função de distribuição acumulada assumida (Normal), e a função de distribuição empírica dos dados. O valor obtido é então comparado com um valor crítico, tabelado conforme o nível de significância adotado (WALPOLE et al., 2009). 
Para dar suporte à suposição da homocedasticidade populacional é comum a aplicação do teste de Bartlett, cuja hipótese de nulidade é a de que as variâncias das populações são homogêneas (GOMES, 1985).

Quando os pressupostos são atendidos e os resultados da ANOVA levam à rejeição da hipótese nula, temos evidências de que pelo menos uma das médias dos tratamentos difere das demais. Entretanto, esta análise não informa qual ou quais são estes tratamentos. Existem vários métodos de comparações múltiplas existentes na literatura que objetivam identificá-los, dentre os quais, podemos citar o teste de Tukey.

O Teste por Tukey (1953) consiste na comparação de todos os possíveis pares de médias e se baseia na diferença mínima (DMS) que deve haver entre duas médias para que elas possam ser consideradas diferentes a um determinado nível de significância adotado (WALPOLE et al., 2009). A hipótese de nulidade do teste de Tukey é a de que não existem diferenças significativas entre os grupos analisados.

A aplicação desses testes de hipóteses em softwares computacionais retorna um valor característico conhecido como valor- $p$. Trata-se da probabilidade de se obter uma estatística de teste maior ou igual a observada na amostra, sob uma hipótese nula. Em termos práticos, aconselha-se a rejeitar a hipótese de nulidade sempre que o valor- $p$ obtido for inferior ao nível de significância adotado.

\section{MATERIAL E MÉTODOS}

Metodologicamente, trata-se de um estudo analítico-descritivo, produzido a partir de dados quantitativos obtidos em bases de dados nacionais, oriundas do Sistema Federal de Inspeção do Trabalho (SFIT) e referentes aos anos compreendidos entre 2010 e 2016.

Inicialmente, foi realizada uma revisão de literatura, pautada nas principais obras e trabalhos recentes e relevantes, associados ao tema, buscando um embasamento teórico para o um bom desenvolvimento do trabalho. Em seguida, efetuou-se a coleta de dados no portal online do SFIT $^{3}$. Nesta fase, devido ao diferente número de trabalhadores em cada setor analisado, considerou-se o número de acidentes por milhão de empregados.

Após a coleta de dados realizou-se uma Análise de Variância para averiguar se existe um setor com média de acidentes diferente dos demais (CECON et al., 2012). Antes, porém, foram verificados os pressupostos para a realização da ANOVA: homocedasticidade (verificada

${ }^{3}$ https://sfitweb.mte.gov.br/ 
por meio do teste de Bartlett), independência (verificada por meio de uma análise gráfica dos resíduos) e normalidade (verificada por meio do teste de Kolmogorov-Smirnov).

Finalmente, foi realizado o teste de comparação múltipla proposto por Tukey (VIEIRA, 2016) para identificar o setor que apresentou maior média de acidentes de trabalho. Em todos os testes de hipóteses realizados, considerou-se 5\% como nível de significância para a interpretação dos resultados.

As análises foram realizadas com o auxílio do software Excel, para organização dos dados em planilhas e do software estatístico R (R CORE TEAM, 2019), por se tratar de um software livre, de código aberto de fácil manipulação.

\section{RESULTADOS E DISCUSSÃO}

As informações coletadas referem-se aos números de trabalhadores e acidentes anuais dos anos de 2010 a 2016, nos setores econômicos de agricultura, construção e indústria. Estas apurações podem ser observadas através das Tabelas 2,3 e 4, em que são apresentados os dados.

Tabela 2 - Acidentes de trabalho na Agricultura

\begin{tabular}{cccc}
\hline Ano & $\begin{array}{c}\text { Trabalhador } \\
\text { es } \\
\text { Alcançados }\end{array}$ & $\begin{array}{c}\text { Acidentes } \\
\text { Analisados }\end{array}$ & $\begin{array}{c}\text { Casos por } \\
\text { Milhão }\end{array}$ \\
\hline 2010 & 1054406 & 76 & 72,078 \\
2011 & 928902 & 99 & 106,577 \\
2012 & 838417 & 100 & 119,272 \\
2013 & 827356 & 89 & 107,572 \\
2014 & 720046 & 100 & 138,88 \\
2015 & 529001 & 107 & 202,268 \\
2016 & 281958 & 51 & 180,878 \\
\hline
\end{tabular}

Fonte: Sistema Federal de Inspeção do Trabalho. 
Tabela 3 - Acidentes de trabalho na Construção Civil

\begin{tabular}{cccc}
\hline Ano & $\begin{array}{c}\text { Trabalhador } \\
\text { es } \\
\text { Alcançados }\end{array}$ & $\begin{array}{c}\text { Acidentes } \\
\text { Analisados }\end{array}$ & $\begin{array}{c}\text { Casos por } \\
\text { Milhão }\end{array}$ \\
\hline 2010 & 2539290 & 526 & 207,145 \\
2011 & 2853439 & 581 & 203,614 \\
2012 & 3490865 & 529 & 151,538 \\
2013 & 3903381 & 634 & 162,423 \\
2014 & 3116431 & 593 & 190,282 \\
2015 & 1957840 & 510 & 260,497 \\
2016 & 985133 & 298 & 302,497 \\
\hline
\end{tabular}

Fonte: Sistema Federal de Inspeção do Trabalho.

Tabela 4 - Acidentes de trabalho na Indústria

\begin{tabular}{cccc}
\hline Ano & $\begin{array}{c}\text { Trabalhador } \\
\text { es } \\
\text { Alcançados }\end{array}$ & $\begin{array}{c}\text { Acidentes } \\
\text { Analisados }\end{array}$ & $\begin{array}{c}\text { Casos por } \\
\text { Milhão }\end{array}$ \\
\hline 2010 & 5136890 & 784 & 152,622 \\
2011 & 5184134 & 709 & 136,762 \\
2012 & 5359208 & 759 & 141,625 \\
2013 & 6920284 & 1011 & 146,092 \\
2014 & 4910261 & 917 & 186,752 \\
2015 & 4199676 & 716 & 170,489 \\
2016 & 2104098 & 402 & 191,056 \\
\hline
\end{tabular}

Fonte: Sistema Federal de Inspeção do Trabalho.

A realização da ANOVA só foi possível depois de verificarmos que os seus pressupostos foram atendidos e a normalidade dos resíduos foi verificada pelo teste de Kolmogorov-Smirnov, cujo valor-p calculado foi 0,7918. A independência dos resíduos foi verificada por meio de uma análise gráfica, conforme indica a Figura 1. 


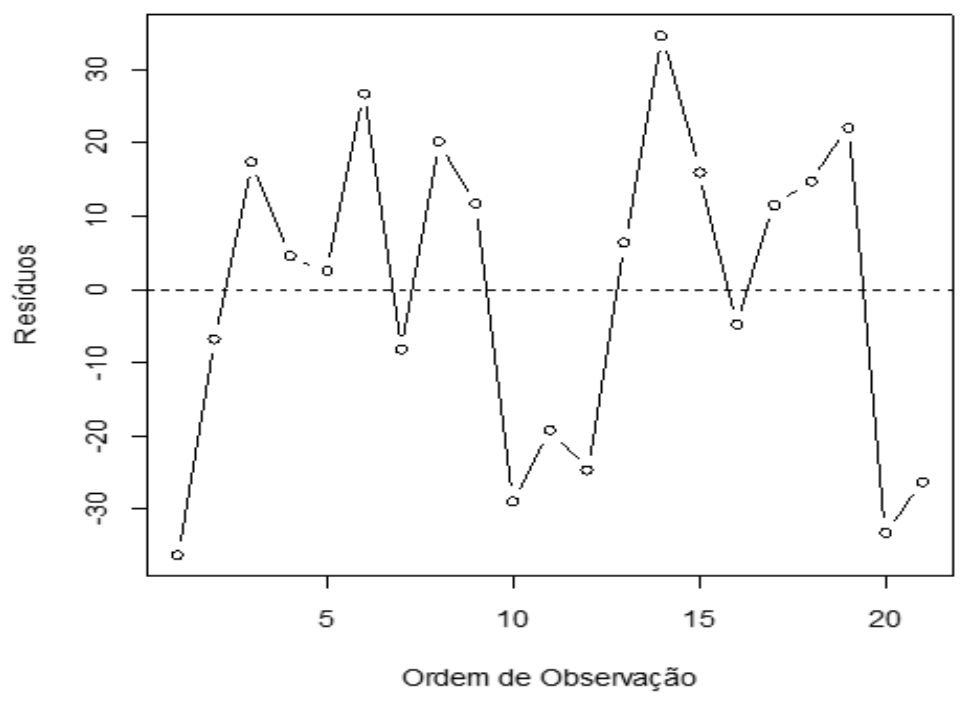

Figura 1 - Análise descritiva da independência dos resíduos

A homocedasticidade foi verificada pelo teste de Bartlett, que apresentou estatística de valor 0,19852 e valor-p 0,9055. Assim, procedeu-se à ANOVA, cujos resultados são apresentados na Tabela 5 .

Tabela 5 - Análise de variância

\begin{tabular}{cccccc}
\hline Fonte & $\begin{array}{c}\text { Graus de } \\
\text { Liberdade }\end{array}$ & $\begin{array}{c}\text { Soma de } \\
\text { Quadrados }\end{array}$ & $\begin{array}{c}\text { Quadrado } \\
\text { Médio }\end{array}$ & $\begin{array}{c}\text { Estatístic } \\
\mathbf{a} \\
\text { F }\end{array}$ & Valor-p \\
\hline Ano & 6 & 23488,60 & 3914,80 & 5,22 & 0.0073913 \\
Setor & 2 & 22213,60 & 11106,80 & 14,80 & 0.0005762 \\
Resíduo & 12 & 9005,80 & 750,50 & & \\
\hline
\end{tabular}

Fonte: Autores (2019).

A ANOVA indicou que, tanto para ano, quanto para o setor (indústria, construção ou agricultura), há pelo menos um grupo diferente dos demais. Dessa forma, procedeu-se ao teste de comparação múltipla de Tukey na busca por melhores interpretações. Nas Tabelas 6 e 7 são apresentados os resultados da comparação, em pares, entre os anos e os setores, respectivamente. 
Tabela 6 - Comparação entre os anos analisados

\begin{tabular}{cccc}
\hline Anos & Valor-p & Anos & Valor-p \\
\hline Ano 1 - Ano 2 & 0.9999835 & Ano 4 - Ano 3 & 1.0000000 \\
Ano 3 - Ano 1 & 0.9999282 & Ano 5 - Ano 3 & 0.7174559 \\
Ano 4 - Ano 1 & 0.9999789 & Ano 4 - Ano 12 & 0.0705348 \\
Ano 5 - Ano 1 & 0.8601637 & Ano 6 - Ano 3 & 0.0255227 \\
Ano 6 - Ano 1 & 0.1124202 & Ano 7 - Ano 3 & 0.7463675 \\
Ano 7 - Ano 1 & 0.0413097 & Ano 5 - Ano 4 & 0.0770771 \\
Ano 3 - Ano 2 & 0.9980807 & Ano 6 - Ano 4 & 0.0279460 \\
Ano 4 - Ano 2 & 0.9989699 & Ano 6 - Ano 5 & 0.6005977 \\
Ano 5 - Ano 2 & 0.9374390 & Ano 7 - Ano 5 & 0.2915213 \\
Ano 6 - Ano 2 & 0.1596798 & Ano 7 - Ano 6 & 0.9949967 \\
Ano 7 - Ano 2 & 0.0599398 & & \\
\hline
\end{tabular}

Fonte: Autores (2019).

Observe que dentre os anos, embora alguns pares apresentassem indícios de diferenças entre as médias à 5\% de significância, uma análise global não permite inferir que há um ano em que as médias de acidentes de trabalho, considerando os três setores analisados, apresentassem diferenças significativas.

Tabela 7 - Comparação entre os setores

\begin{tabular}{cc}
\hline Área & Valor-p \\
\hline Construção - Agricultura & 0.0004558 \\
Indústria - Agricultura & 0.1725848 \\
Indústria - Construção & 0.0125857 \\
\hline
\end{tabular}

Fonte: Autores (2019).

A análise da Tabela 7 nos permite concluir que existe indícios de uma diferença tanto entre a construção e a agricultura, quanto entre a indústria e a construção, à 5\% de significância. Para verificar qual apresenta maiores médias foi construída a Figura 2. 


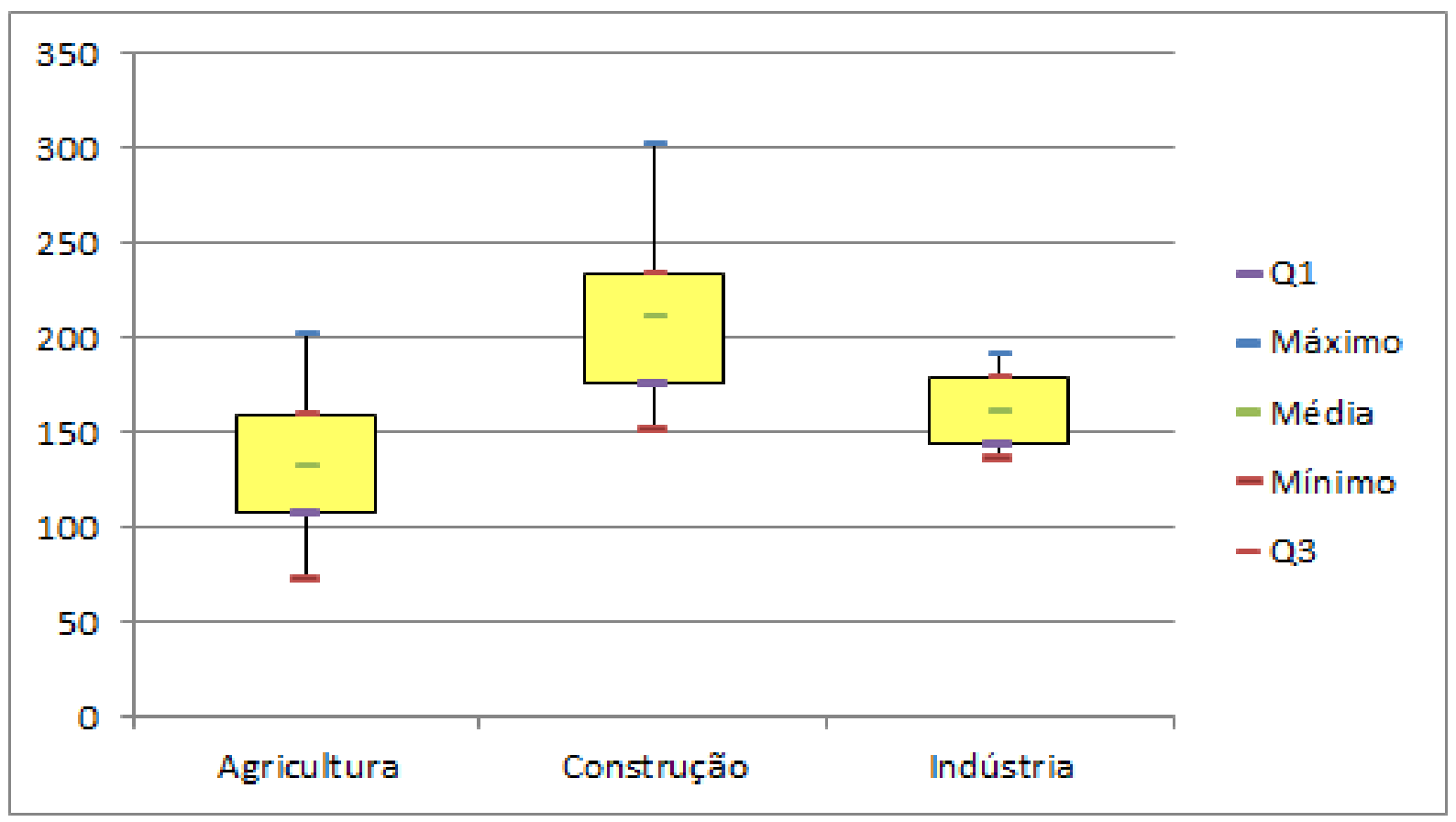

Figura 2 - Boxplot relacionando cada setor com o número de acidentes por milhão Fonte: Autores (2019).

Verifica-se, pela análise gráfica que a construção civil foi o setor que apresentou maior média de acidentes, dentre os setores no período analisados. A média de acidentes dos setores da Agricultura e da Indústria não apresentaram diferenças estatisticamente significativas.

\section{CONCLUSÕES}

Este artigo teve o intuito de apresentar os índices de acidentes de trabalho em três dos principais setores da economia: agricultura, indústria e construção civil e de propor uma discussão acerca da temática. Esses acidentes são frequentes e é preciso um investimento contínuo em prevenção, por meio de um plano de ações que possa auxiliar a gestão das empresas no âmbito da segurança do trabalho.

As análises estatísticas apontaram o setor da construção civil como o de maiores médias anuais de acidentes de trabalho, apontando para a necessidade de um estudo mais aprofundado sobre os índices deste setor cujos trabalhadores desempenham diversificadas atividades que os expõem a riscos de acidentes, a todo momento.

[...] A construção civil possui características singulares: é uma indústria nômade; cria produtos únicos; utiliza mão de obra de baixa qualidade; envolve grandes quantidades de insumos; é uma indústria muito tradicional apresentando grande inércia às inovações; o grau de precisão de trabalho é menor do que em outras indústrias; realiza trabalhos sob intempéries e sofre acentuada pressão de tempo devido aos cronogramas. Estes fatores implicam na dificuldade para o gerenciamento do empreendimento e, principalmente, para o gerenciamento dos riscos destes 
empreendimentos e, em especial, para o gerenciamento de riscos com quedas (CBIC, 2019, p. 96).

Este trabalho, de caráter inicial, não tem a pretensão de apontar apenas a necessidade de medidas preventivas à acidentes de trabalho na construção civil. Embora o setor tenha se destacado como o que apresenta maiores médias, tanto a agricultura, quanto a indústria também apresentam números alarmantes.

Acredita-se que um investimento em segurança no trabalho tem impactos sociais e econômicos de grande representatividade no mercado. Assim, espera-se que este trabalho seja um instrumento de difusão de sua importância e promova debates sobre a temática aqui apresentada, abrindo caminhos para novos estudos e pesquisas relacionadas a este tema.

\section{REFERÊNCIAS}

BRASIL. Lei $n^{\circ}$ 8.213, de 24 de julho de 1991. Dispõe sobre os planos de benefícios da previdência social e dá outras providências. Disponível em:

http://www.planalto.gov.br/ccivil_03/Leis/L8213cons.htm Acesso em 13 de ago. 2019.

BUSSAB, W. O.; MORETTIN, P. A. Estatística básica. 6. ed. São Paulo: Saraiva, São Paulo: Saraiva, 2013.

CBIC, 2019. Segurança e saúde na indústria da construção: prevenção e inovação. Câmara Brasileira da Indústria da Construção. Brasília: CBIC, 2019. Disponível em:

https://cbic.org.br/wp-

content/uploads/2019/04/SEGURANCA_E_SAUDE_NA_INDUSTRIA_DA_CONSTRUCA

O_Prevencao_e_Inovacao.pdf Acesso em 13 de agosto de 2019.

CECON, P. R. et al. Métodos estatísticos. Viçosa: UFV, 2012.

CESTEH. Brasil é um dos países com maior número de mortes e acidentes de trabalho no mundo. Será o trabalhador brasileiro superprotegido? [s.l]. 2019. Disponível em: http://www.cesteh.ensp.fiocruz.br/noticias/brasil-e-um-dos-paises-com-maior-numero-demortes-e-acidentes-de-trabalho-no-mundo-sera-o Acesso em: 13 ago. 2019.

CHIAVENATO, I. Recursos humanos. 7. ed. São Paulo: Atlas, 2002.

COELHO, D. F. B.; GHISI, B. M.; Acidentes de trabalho na construção civil, p. 23 -32. In: Acidente de trabalho na construção civil em Rondônia. São Paulo: Blucher, 2016.

GOMES, F. P. Curso de estatística experimental. Piracicaba: ESALQ/USP, 1985.

OLIVEIRA, S. G. Proteção jurídica à saúde do trabalhador. 2. ed. São Paulo: LTr, 1998.

SOUSA, F. N. F.; SANTANA, V. S. Mortalidade por acidentes de trabalho entre

trabalhadores da agropecuária no Brasil, 2000-2010. Rio de Janeiro, p.2-2, 24 jul. 2015. 
Disponível em: http://www.scielo.br/pdf/csp/v32n4/1678-4464-csp-32-04-e00071914.pdf. Acesso em: 22 nov. 2018.

R DEVELOPMENT CORE TEAM. R: A Language and environment for statistical computing. Vienna: R Foundation on Statistical Computing, 2019. Disponível em: https://www.r-project.org. Acesso: 13/08/2019.

TUKEY, J. W. Section of mathematics and engineering: some selected quick and easy methods of statistical analysis. Transactions of the New York Academy of Sciences, v. 16, n. 2 p. 88-97, 1953.

VIEIRA, S. Teste de Tukey para comparação de médias, 2016. Disponível em: http://soniavieira.blogspot.com/2016/06/paraproceder-ao-teste-de-tukey-e.html. Acesso em: 26 out. 2018.

WALPOLE, R. E. et al. Probabilidade e estatística para engenharia e ciências. São Paulo: Pearson, 2009.

\section{DADOS DOS AUTORES}

\section{Nome: Ketelin Rúbia Almeida Silva}

E-mail: ketelin.rubia.almeida@gmail.com

Currículo Lattes: http://lattes.cnpq.br/7492586989820502

Graduanda em Bacharelado em Engenharia Civil no Instituto Federal de Educação, Ciência e Tecnologia de Minas Gerais (IFMG) - Campus Piumhi.

\section{Nome: Júlia Silva de Jesus}

E-mail: julliasilva.lp@gmail.com

Currículo Lattes: http://lattes.cnpq.br/7352537507004952

Graduanda em Bacharelado em Engenharia Civil no Instituto Federal de Educação, Ciência e Tecnologia de Minas Gerais (IFMG) - Campus Piumhi.

\section{Nome: André Henrique Garcia Silva}

E-mail: andreengcivil10@gmail.com

Currículo Lattes: http://lattes.cnpq.br/1126132343731052

Graduando em Bacharelado em Engenharia Civil no Instituto Federal de Educação, Ciência e Tecnologia de Minas Gerais (IFMG) - Campus Piumhi e, atualmente é estagiário de obra de pavimentação e rodovias da Pavidez Engenharia LTDA.

\section{Nome: Talita Emidio Andrade Soares}

E-mail: talitaeandrade@gmail.com

Currículo Lattes: http://lattes.cnpq.br/0130349523076322

Mestranda do Programa de Pós-Graduação em Educação da Universidade Federal do Espírito Santo (UFES). Licenciada em Matemática pela Universidade Federal de Viçosa (UFV). Professora substituta do Instituto Federal de Minas Gerais (IFMG) - Campus Bambuí. É membro do Grupo de Pesquisa Educação Matemática e Educação Profissional (EMEP-IFES) e do Instituto de Pesquisa em Educação e Educação Física (PROTEORIA-UFES).

\section{Nome: Denilson Junio Marques Soares}

E-mail: denilson.marques@ifmg.edu.br

Currículo Lattes: http://lattes.cnpq.br/1813099481556666 
Doutorando do Programa de Pós-Graduação em Educação da Universidade Federal do Espírito Santo (UFES). Mestre em Estatística Aplicada e Biometria e Licenciado em matemática pela Universidade Federal de Viçosa (UFV). Professor EBTT do Instituto Federal de Educação, Ciência e Tecnologia de Minas Gerais (IFMG) - Campus Piumhi. É membro do grupo de pesquisa Educação Matemática e Educação Profissional (EMEP-IFES) e do Instituto de Pesquisa em Educação e Educação Física (PROTEORIA-UFES). 